\title{
Coagulopatía, tratamiento anticoagulante en COVID-19 y trombosis posvacunación
}

Carlos Martínez-Murillo ${ }^{*}$ y Ángel G. Vargas-Ruiz²

${ }^{1}$ Departamento de Hematología, Hospital General de México Dr. Eduardo Liceaga; ²Departamento de Hematología y Oncología, Instituto Nacional de Ciencias Médicas y Nutrición Salvador Zubirán. Ciudad de México, México

\section{Resumen}

La coagulopatía y la trombosis son situaciones graves que afectan a los pacientes con enfermedad por coronavirus 2019 (COVID-19) que requieren hospitalización. En estos pacientes se alteran mecanismos procoagulantes y fibrinolíticos que condicionan un estado procoagulante progresivo y grave. La anticoagulación oportuna en estos pacientes es importante, pero han surgido preguntas sobre el tipo, la dosis y el momento adecuado de la anticoagulación. Las directrices y documentos de consenso existentes ofrecen sugerencias generales sobre la dosis de heparinas de bajo peso molecular en función de la gravedad de la enfermedad y el riesgo de trombosis, pero todavía falta una relación entre los marcadores de coagulación y el régimen de anticoagulación. Se están llevando a cabo muchos ensayos clínicos que abordan estas cuestiones; se alienta la participación en estos ensayos para determinar las mejores estrategias de tratamiento para los pacientes de COVID-19. Es necesario aumentar los conocimientos con un rápido intercambio para atender adecuadamente a los pacientes en esta pandemia.

PALABRAS CLAVE: Coagulopatía. Trombosis. Coagulación. Anticoagulación. Heparinas. COVID-19.

\section{Coagulopathy, anticoagulant treatment in COVID-19 and thrombosis postvaccine}

\begin{abstract}
Coagulopathy and thrombosis are serious situations that COVID-19 patients require hospitalization. In these patients, procoagulant and fibrinolytic mechanisms are altered that condition a progressive and severe procoagulant state. Timely anticoagulation in these patients is important, but questions have been raised about the type, dose, and timing of anticoagulation. The guidelines and consensus documents offer general suggestions on the dose of LMWH based on the severity of the disease and the risk of thrombosis, but a relationship between coagulation markers and anticoagulation regimen is still lacking. Many clinical trials are underway that address these issues; Participation in these trials to determine the best treatment strategies for COVID-19 patients is encouraged. Increasing knowledge with rapid exchange is necessary to adequately care for patients in this pandemic.
\end{abstract}

KEY WORDS: Coagulopathy. Thrombosis. Coagulation. Anticoagulation. Heparins. COVID-19.

Correspondencia:

${ }^{*}$ Carlos Martínez-Murillo

E-mail: carlosmtzmurillo@gmail.com
Gac Med Mex. 2021;157(Supl 3):S79-S89

Disponible en PubMed

www.gacetamedicademexico.com

0016-3813/@ 2021 Academia Nacional de Medicina de México, A.C. Publicado por Permanyer. Este es un artículo open access bajo la licencia CC BY-NC-ND (http://creativecommons.org/licenses/by-nc-nd/4.0/). 


\section{Introducción}

La Organización Mundial de la Salud clasificó el brote de enfermedad por coronavirus 2019 (COVID-19) como una pandemia y la enfermedad contribuyó a una mortalidad significativa en todo el mundo, explicada principalmente por el síndrome de dificultad respiratoria aguda fatal. Esta morbilidad y mortalidad secundaria a COVID-19 está aumentando en todo el mundo. La tromboembolia venosa (TEV) (incluida la embolia pulmonar), la trombosis arterial y los trombos microvasculares parecen contribuir a los resultados adversos. Sin embargo, el daño pulmonar no es la única razón del alto riesgo de muerte; a pesar de la profilaxis anticoagulante, varias unidades de cuidados intensivos ( $\mathrm{UCl}$ ) informaron trombosis arterial $y$ venosa potencialmente mortal, en particular embolias pulmonares graves frecuentes ${ }^{1-4}$.

Estas observaciones clínicas condujeron al tratamiento empírico de pacientes hospitalizados con COVID-19 con heparina de bajo peso molecular (HBPM) en dosis más altas que la tromboprofilaxis habitual ${ }^{1}$. Sin embargo, algunos pacientes aún exhibieron complicaciones trombóticas mientras estaban en profilaxis con dosis altas ${ }^{5}$. También se demostró que los dímeros $D$ altos $>3,000 \mathrm{ng} / \mathrm{ml}$ constituían un biomarcador para el mal pronóstico de la enfermedad ${ }^{6}$.

La fisiopatología de la trombosis relacionada con COVID-19 no se comprende completamente. Se han informado niveles elevados de fibrinógeno, factor VIII (FVIII), factor de von Willebrand (FvW), anticoagulante lúpico (AL) positivo y anticuerpos antifosfolípidos ${ }^{7}$. La tormenta de citocinas asociada con COVID-19 probablemente tiene un impacto importante en los niveles plasmáticos de las proteínas de coagulación mencionadas anteriormente, creando un cambio sustancial en el equilibrio entre las actividades procoagulantes $y$ anticoagulantes.

Como consecuencia, los informes patológicos mostraron depósitos de fibrina dentro del pulmón ${ }^{8-12}$.

La discrepancia entre la frecuencia de complicaciones trombóticas y la intensidad de la profilaxis antitrombótica administrada a pacientes con COVID-19 es intrigante. Parece ser que las anormalidades fibrinolíticas asociadas con el aumento de la generación de trombina podrían ser contribuyentes clave para la trombosis inducida por COVID-19.

Así, a pesar de los sustanciales niveles de plasma de heparina, la generación de trombina en algunos pacientes puede ser normal, lo que sugiere una importante hipercoagulabilidad que no pudo ser controlada con la terapia de heparina o una resistencia a la heparina. Por otra parte, los niveles de fibrinógeno y del FVIII se encuentran significativamente elevados en todos los pacientes de COVID-19, lo que apoya la hipótesis de una hipercoagulabilidad no controlada, probablemente relacionada con un síndrome inflamatorio mayor.

Además de una generación de trombina muy alta se observa fibrinólisis deteriorada en todos los pacientes de COVID-19. Los pacientes de la UCl que presentan una forma grave de la enfermedad tienen niveles significativamente más altos de activador del plasminógeno tisular (tPA), inhibidor del activador del plasminógeno-1 (PAI-1) y del inhibidor fibrinolítico activable por trombina (TAFI) a/i en comparación con los pacientes que no estaban en la UCl. El estado hipercoagulable existente en la infección por coronavirus 2 del síndrome respiratorio agudo grave (SARSCoV-2) puede dar lugar a la generación de altas concentraciones de trombina necesarias para activar el TAFI. Se ha demostrado que el aumento de los niveles de antígeno del TAFI y se asocia con riesgo de trombosis arterial ${ }^{9,10}$. A pesar de la corta vida media de la TAFla, los datos de modelos animales y estudios clínicos indicaron que la cantidad de TAFla podría desempeñar un papel más crucial en el retraso de la fibrinólisis que la cantidad total de proteína $\mathrm{TAFI}^{11,12}$.

La inflamación promueve la liberación local de tPA y PAl-1 de las células endoteliales12. Además, las plaquetas activadas también pueden liberar grandes cantidades de PAI-1, ya que las plaquetas son el principal pool circulante de PAI-1 que puede contribuir a una alta concentración local de PAI-1 en el sitio de un coágulo de fibrina en crecimiento. El aumento del PAl-1 es responsable de la hipofibrinólisis y la persistencia de la fibrina. La deposición persistente de fibrina en el parénquima pulmonar y los espacios alveolares de los pacientes de COVID-19 indica claramente que, a pesar del aumento de los niveles de tPA, los altos niveles de PAI-1 pueden superar la liberación local de tPA. Además, la hipofibrinólisis plasmática debida a niveles elevados de PAI-1 y TAFI es un factor de riesgo de trombosis venosa12. Se puede pensar que los altos niveles plasmáticos de PAl-1 encontrados en pacientes de la $\mathrm{UCl}$ pueden explicarse por la alta incidencia de infección grave por COVID-19 en obesos debido a que el tejido adiposo contribuye a la producción de PAI-13. 
La tromboelastografía es un ensayo de hemostasia global, capaz de evaluar simultáneamente los componentes de coagulación y fibrinolíticos ${ }^{14}$. Este ensayo de sangre total es fácil de realizar, rápido, con reactivos estandarizados, y está disponible en un número cada vez mayor de laboratorios de hematología. El ensayo se utiliza habitualmente para evaluar la coagulación y puede detectar la hiperfibrinólisis en pacientes con traumatismos. En trabajos recientes se informó de que los pacientes de COVID-19 presentan una hipercoagulabilidad severa en lugar de una coagulopatía consuntiva mediante tromboelastografía, pero en esos estudios no se informó de ninguna anomalía de la fibrinólisis ${ }^{15,16}$.

Una de las intervenciones clave que parece ser eficaz para reducir la mortalidad asociada a la trombosis en entornos sin COVID-19 es el tratamiento anticoagulante. En consecuencia, la Sociedad Internacional de Trombosis y Hemostasia (ISTH) publicó una directriz provisional que recomendaba el uso de anticoagulación profiláctica con heparina de bajo peso molecular (HBPM) en todos los pacientes ingresados con COVID-19 en ausencia de contraindicaciones $^{4}$. Aunque se sugieren posibles soluciones, subrayamos la necesidad de realizar estudios aleatorizados bien diseñados (realizados rápidamente) para producir recomendaciones basadas en la evidencia. Cabe señalar que las siguientes recomendaciones son solo para pacientes adultos ${ }^{17}$.

\section{Evidencia de trombosis en COVID-19}

Los estudios en diferentes países han mostrado que la enfermedad COVID-19 severa es una clara condición protrombótica, que aumenta el riesgo de trombosis, en particular las que son venosas.

En un estudio multicéntrico se reunió a una cohorte prospectiva de 150 pacientes con COVID-19 severo de cuatro unidades de terapia intensiva en Francia, 122 eran hombres, con edad media de 63 años (rango de 53 a 71 años). La mayoría de los pacientes (95\%) tuvo elevaciones importantes de dímero $\mathrm{D}$, de fibrinógeno, FVIII y FvW. No hubo pacientes con trombocitopenia y los niveles de antitrombina (AT) fueron normales. Los tiempos de coagulación no se prolongaron en forma importante. Un $87.7 \%$ tuvieron anticoagulante lúpico $(A L)$ positivo. Ningún paciente tuvo CID de acuerdo con el score de la ISTH. Veintisiete de estos pacientes tuvieron trombosis, esto es un $18 \%$. Cuando estos pacientes se compararon con una cohorte de pacientes con falla respiratoria de otra causa, no COVID-19, se vio que las trombosis son 3.4 veces más frecuentes entre los pacientes con COVID19 severo, principalmente las trombosis venosas, tromboembolia pulmonar (TEP). Una observación que hicieron es que los pacientes que ocuparon hemodiálisis por insuficiencia renal aguda tuvieron significativamente más oclusiones trombóticas del circuito. La incidencia de hemorragia entre los pacientes con COVID-19 fue muy baja (2.7\%) y no fue estadísticamente diferente de la incidencia en los pacientes sin COVID-19.18.

En otras series se ha encontrado una incidencia mucho más alta de trombosis. En un estudio se reunió a 143 pacientes con COVD-19 que fueron hospitalizados en Wuhan, China. La edad media de los pacientes fue de 63 años, la mitad hombres. 66 pacientes desarrollaron TVP (46.1\%), la tercera parte (34\%) fueron TVP proximales y las dos terceras partes restantes fueron distales. Solo la tercera parte de estos pacientes con TVP estaba bajo profilaxis, la mayoría no tenía ningún anticoagulante. Se observó también que los pacientes con TVP tienen una menor supervivencia en comparación a los que no hacen trombosis. En el análisis multivariante, los tres factores relacionados a trombosis fueron la presencia de insuficiencia respiratoria, la presencia de comorbilidades (medida con el score de Padua mayor o igual de 4) y un dímero $D$ elevado (mayor de 1,000 $\mu \mathrm{g} / \mathrm{l}^{19}$.

La trombosis en COVID-19 es más prevalente en los pacientes mas graves, los que están en la $\mathrm{UCl}, \mathrm{y}$ esto tiene un impacto directo en la supervivencia. En un estudio de 198 pacientes hospitalizados con COVID-19 en un solo centro en Holanda, 75 pacientes (38\%) estaban en la $\mathrm{UCl}$, el resto en la sala de medicina interna y tras una mediana de seguimiento de siete días, se encontró TEV en 39 pacientes (20\%). La incidencia de TEV fue 6 veces más alta en pacientes de la $\mathrm{UCl}$ (59 vs. $9.2 \%$ a 21 días). Los pacientes con TEV tuvieron 2.4 veces más riesgo de morir ${ }^{20}$.

\section{El uso de anticoagulantes en COVID-19}

Hay pocas cosas que han demostrado mejorar la supervivencia de los pacientes con COVID-19, y una de ellas es la anticoagulación ${ }^{2}$. En un estudio se comparó la mortalidad a 28 días entre pacientes con COVID-19 severo que usaron heparina y los que no. Fueron 449 pacientes, 99 de los cuales recibieron heparina (principalmente HBPM) a dosis profiláctica. La mortalidad a 28 días no fue diferente entre los que usaron heparina y los que no, para el grupo en 
general (30.3\% en los que usaron heparina vs. $29.7 \%$ entre los que no, sin diferencia significativa). Pero donde la heparina sí tuvo utilidad fue en los pacientes más graves, con SIC (sepsis-induced coagulopathy) score mayor o igual a 4 y aquellos con dímero $D$ de más de $3 \mu \mathrm{g} / \mathrm{ml}$ que tuvieron menor mortalidad con 32 vs. $52 \%(p=0.017)^{2}$.

Hasta el momento no existen estudios prospectivos que evalúen el uso de la anticoagulación terapéutica con heparina en los pacientes con COVID-19. Al menos 18 estudios clínicos prospectivos están registrados en Clinical Trials, de los cuales nueve se encuentran reclutando pacientes, pero al momento de esta publicación no tenemos resultados de ninguno. Por ahora, solo contamos con la evidencia de estudios retrospectivos como el realizado en el Hospital Monte Sinaí en Nueva York, EE.UU. Entre marzo y abril de 2020 ellos reunieron a 2,773 pacientes hospitalizados por COVID-19. De estos, 786 pacientes $(28 \%)$ recibieron anticoagulación durante su hospitalización. Para el grupo en general, al día 21 la mortalidad fue del $22.5 \%$ en los pacientes anticoagulados vs. $22.8 \%$ en los no anticoagulados, sin diferencia estadísticamente significativa. Sin embargo, cuando solo se analizaron los pacientes más graves, los que tenían insuficiencia respiratoria con ventilación mecánica invasiva, la mortalidad a 21 días fue del $29 \%$ para los que recibieron anticoagulación vs. $62 \%$ para los que no la recibieron. La mediana de supervivencia fue de nueve días para los que no recibieron anticoagulación vs. 21 días para los que sí la recibieron ${ }^{5}$. Esta es una clara evidencia a favor del uso de anticoagulantes, sin embargo, debe tenerse precaución con el sangrado, ya que el sangrado mayor ocurrió en el $7.5 \%$ para los pacientes graves anticoagulados vs. $1.3 \%$ para los que no se anticoagularon. En otras series de pacientes COVID-19 con anticoagulación se ha reportado un $4.8 \%$ de eventos de sangrado, con un $2.3 \%$ de eventos de sangrado mayor, la mayoría en pacientes graves. Los sangrados fueron digestivos o hemoptisis. El dímero D alto, la trombocitopenia, la hipofibrinogenemia y el ser paciente grave en la $\mathrm{UCl}$ fueron los factores de riesgo significativos para hemorragia en los pacientes COVID-19 anticoagulados con heparina ${ }^{5,6}$.

Lo anterior significa que la coagulopatía por COVID19 no es primariamente hemorrágica, pero que a medida que los pacientes se agravan, y sobre todo si se usa heparina, la incidencia de sangrado aumenta, lo que debe tenerse presente siempre que usemos heparinas en los pacientes graves.
Algunas publicaciones han demostrado ya que la heparina tiene además de sus efectos anticoagulantes, efectos antiinflamatorios y de protección endotelial y que hasta puede mejorar el funcionamiento pulmonar y la función cardiaca ${ }^{21}$.

Entre los mecanismos antiinflamatorios descritos están: la unión a citocinas inflamatorias, la inhibición de la quimiotaxis y la migración de leucocitos, principalmente neutrófilos, inhibición del complemento (C5a), secuestro de proteínas reactantes de fase aguda, unión a las histonas y a patrones moleculares asociados a daño, protección de la célula endotelial con disminución del edema e inhibición de las vías MAPK y de factor nuclear kappa $B$ en la célula endotelial ${ }^{22}$.

Las heparinas también tienen un efecto antiviral, el cual ha sido estudiado en modelos experimentales. Por su naturaleza polianiónica, su carga negativa y su alta sulfatación, la heparina puede unirse a proteínas de la superficie endotelial y prevenir o evitar la unión de los virus. Se ha demostrado que la heparina interactúa con la proteína S1 del SARS-CoV-2 y en líneas celulares experimentales la heparina puede reducir la infección de las células.

Las heparinas son polisacáridos, glucosaminoglicanos sulfatados que comercialmente se obtienen de mucosa intestinal porcina o del pulmón bovino, ricos en mastocitos. Las heparinas toman el papel del heparán sulfato, un proteoglicano de la pared celular de las células de los mamíferos, que se une a la antitrombina para favorecer la inactivación de los factores Ila y Xa de la coagulación. La heparina no fraccionada (HNF) tiene un peso molecular entre 5,000 y 30,000 dalton, las HBPM tienen un peso molecular de 3,000 a 6,500 dalton. Mientras más grande y sulfatada es la cadena de sacáridos, las heparinas tienen una mayor actividad para inhibir a la trombina, más es también su unión a proteínas y células, por lo que su vida media es corta, su depuración es celular y hay más riesgo de trombocitopenia inducida por heparina (HIT). Mientras más corta es la cadena, las heparinas pierden actividad anti-lla conservando solo la actividad anti-Xa, su vida media es más prolongada, la depuración es más renal y causan menos HIT. El pentasacárido, fondaparinux, como su nombre lo dice, solo tiene la pequeña secuencia básica que se une a la AT y su efecto por ello es exclusivo anti-Xa. Las dosis convencionales, así como los ajustes de las heparinas, se muestran en la tabla 1.

Las interacciones farmacológicas de los agentes antitrombóticos deben ser consideradas, particularmente si 
Tabla 1. Dosis profilácticas y terapéuticas de las heparinas. Ajuste en obesidad e insuficiencia renal

\begin{tabular}{|c|c|c|c|}
\hline Agente & Dosis & Dosis en obesidad & Ajuste en insuficiencia renal \\
\hline Enoxaparina & $\begin{array}{l}1 \mathrm{mg} / \mathrm{kg} / 12 \mathrm{~h} \text { para tratamiento } \\
40 \mathrm{mg} \text { al día para profilaxis }\end{array}$ & $\begin{array}{l}1 \mathrm{mg} / \mathrm{kg} / 12 \mathrm{~h} \text { para tratamiento } \\
40 \mathrm{a} 60 \mathrm{mg} \text { cada } 12 \mathrm{~h} \text { para profilaxis }\end{array}$ & $\begin{array}{l}\mathrm{DCr}<30 \mathrm{ml} / \mathrm{min} \text { reducir a } 1 \mathrm{mg} / \mathrm{kg} / \text { día en } \\
\text { tratamiento y a } 30 \mathrm{mg} \text { diarios para profilaxis }\end{array}$ \\
\hline Dalteparina & $\begin{array}{l}200 \text { UI/kg/día para tratamiento } \\
5,000 \text { U al día para profilaxis }\end{array}$ & $\begin{array}{l}200 \text { UI/kg/día para tratamiento } \\
7,500 \text { U al día para profilaxis }\end{array}$ & $\begin{array}{l}\text { No se recomienda su uso con } \\
\mathrm{DCr}<30 \mathrm{ml} / \mathrm{min}\end{array}$ \\
\hline Tinzaparina & $\begin{array}{l}175 \mathrm{Ul} / \mathrm{kg} / \text { día para tratamiento } \\
4,500 \mathrm{U} \text { al día para profilaxis }\end{array}$ & $\begin{array}{l}175 \text { Ul/kg/día para tratamiento } \\
<110 \mathrm{~kg} \mathrm{4,500} \mathrm{U} \mathrm{al} \mathrm{día} \\
>160 \mathrm{~kg} \mathrm{14,000} \mathrm{U} \mathrm{al} \mathrm{día}\end{array}$ & No requiere ajuste \\
\hline Nadroparina & $\begin{array}{l}86 \mathrm{UI} / \mathrm{kg} / 12 \mathrm{~h} \text { o } 171 \mathrm{UI} / \mathrm{kg} / \text { día para } \\
\text { tratamiento } \\
3,800 \mathrm{UI} \text { al día para profilaxis }\end{array}$ & $\begin{array}{l}86 \text { Ul/kg/12 h o } 171 \text { UI/kg/día para } \\
\text { tratamiento } \\
5,700 \mathrm{U} \text { al día para profilaxis }\end{array}$ & $\begin{array}{l}\mathrm{DCr}<50 \mathrm{ml} / \mathrm{min} \text { reducir } 25 \text { a } 33 \% \text { la dosis } \\
\text { profiláctica o terapéutica } \\
\mathrm{DCr}<30 \mathrm{ml} / \mathrm{min} \text { no se recomienda uso } \\
\text { terapéutico }\end{array}$ \\
\hline Fondaparinux & $\begin{array}{l}7.5 \mathrm{mg} / \text { día para tratamiento } \\
2.5 \mathrm{mg} \text { al día para profilaxis }\end{array}$ & $\begin{array}{l}10 \mathrm{mg} / \text { día para tratamiento } \\
5 \mathrm{mg} \text { al día para profilaxis }\end{array}$ & No debe usarse \\
\hline $\begin{array}{l}\text { Heparina no } \\
\text { fraccionada }\end{array}$ & $\begin{array}{l}80 \text { UI/kg en bolo IV, seguido de } 18 \\
\text { UI/kg/h IV para tratamiento } \\
5,000 \text { U SC cada } 8-12 \text { h para } \\
\text { profilaxis }\end{array}$ & $\begin{array}{l}80 \text { UI/kg en bolo IV, seguido de } 18 \\
\text { UI/kg/h IV para tratamiento } \\
7,500 \text { UI SC cada } 8 \text { h para profilaxis }\end{array}$ & No requiere ajuste \\
\hline
\end{tabular}

el paciente con COVID-19 recibe algún tipo de terapia antiviral o antiinflamatoria. Ni los anticoagulantes antivitamina $\mathrm{K}(\mathrm{AVK})$ ni los anticoagulantes de acción directa (DOAC) se recomiendan para el manejo hospitalario de los pacientes con COVID-19 por sus importantes interacciones farmacológicas. Por ejemplo, los niveles plasmáticos de los DOAC se aumentan hasta seis o siete veces cuando los pacientes son tratados en forma concomitante con fármacos antivirales. Con base en esto, se recomienda que los pacientes que antes de hospitalizarse recibían DOAC o AVK, durante su internamiento por COVID-19 deben cambiar a una heparina ${ }^{23-25}$.

\section{Recomendaciones para el manejo anticoagulante en los pacientes con COVID-19}

Se han publicado múltiples guías y lineamientos para el manejo de la anticoagulación en los pacientes con COVID-19.

\section{Pacientes con COVID-19 leve o asintomático}

Los pacientes con formas leves no requieren anticoagulación. Deben mantenerse en aislamiento en casa, favoreciendo en lo posible la deambulación. Solo en el caso de pacientes con riesgo tromboembólico alto (como aquellos con trombosis previa o con obesidad mórbida), sin riesgo de sangrado, pudiera valorarse el uso de tromboprofilaxis. Si el paciente usa anticoagulantes 0 antiplaquetarios por alguna condición previa al COVID-19, se debe continuar con este manejo. Al respecto de los pacientes que antes de contraer COVID-19 ya usaban anticoagulantes o antiagregantes se ha investigado si el uso de estos antitrombóticos protege contra el riesgo de presentar insuficiencia respiratoria o de morir a causa de COVID19 y se ha encontrado que el usar antitrombóticos no protege contra desenlaces fatales por COVID-19 grave $^{26}$. Los pacientes con enfermedades crónicas como hipertensión, diabetes, cardiopatías, etc., deben continuar con sus tratamientos y en lo posible tener el control óptimo de su patología.

\section{Pacientes con COVID-19 hospitalizados en la sala de medicina interna}

Para todos los pacientes hospitalizados, estén críticos o no, la recomendación es que reciban tromboprofilaxis de acuerdo con su riesgo de TEV. El riesgo de trombosis se determina usando las escalas que ya han sido validadas para este fin, como IMPROVE o Padua. La mayoría de los pacientes con COVID 19 que son hospitalizados tienen comorbilidades que los vuelven pacientes de riesgo alto para trombosis, por lo que las diferentes guías recomiendan la tromboprofilaxis con 
HBPM una vez al día o heparina convencional dos o tres veces al día. También debe valorarse el riesgo de sangrado y si hay contraindicaciones para la tromboprofilaxis con heparinas (como el sangrado activo 0 la trombocitopenia severa), debe ofrecerse profilaxis mecánica. La profilaxis con heparinas debe ser ajustada a peso y a la función renal.

Todas las guías están de acuerdo en que los pacientes hospitalizados con COVID-19 deben recibir HNF o HBPM a dosis profiláctica ${ }^{17-23}$, pero algunas guías recomiendan dosis más altas de HBPM o HNF que se han denominado «dosis intermedias» para los pacientes más graves, con dímero $\mathrm{D}$ muy alto (arriba de seis veces lo normal), troponina muy elevada (arriba de $20 \mathrm{ng} / \mathrm{ml}$ ), ferritinas muy altas (arriba de $1,500 \mathrm{ng} / \mathrm{ml}$ ) o en los pacientes con obesidad mórbida. Esta recomendación se basa en las observaciones clínicas de pacientes que presentan trombosis aun sobre las dosis profilácticas de heparina. En cualquier caso, es importante puntualizar que el uso de dosis de HBPM diferentes a las profilácticas no tiene por ahora el soporte de algún estudio o publicación y solo tiene bases pragmáticas. Las dosis intermedias para las heparinas más usuales en nuestro medio están en la tabla 1.

\section{Pacientes con COVID-19 severa en terapia intensiva}

Los pacientes con COVID-19 severa que están en la $\mathrm{UCl}$ son los que tienen el pronóstico más adverso, con una mortalidad del $42 \%$, sobre todo si se trata de pacientes con comorbilidades, SIC score mayor 0 igual a 4, dímero $D$ muy alto (arriba de 6 veces el limite normal), marcadores altos de inflamación, etc.

Para los pacientes en la UCI, con COVID-19 moderado o severo las guías recomiendan también HBPM a dosis profilácticas, aunque la estrategia óptima de tromboprofilaxis se desconoce aún. Para los pacientes con coagulopatía más severa, con incremento progresivo del dímero $D$, inflamación severa, datos de insuficiencia renal o hepática o en inm i n e n t e insuficiencia respiratoria, dosis intermedias o terapéuticas de HBPM deberían ser consideradas, de acuerdo con el riesgo de sangrado. Los pacientes con obesidad también deben ser llevados a dosis más altas de heparina (Tablas 2-4).

Los pacientes con stent coronario que requieran doble terapia con antiplaquetarios podrán continuar con ellos si las plaquetas son superiores a 50,000/ul, usando solo aspirina si las plaquetas están entre 25,000 y $50,000 / \mu l$ y suspendiendo los antiplaquetarios si las plaquetas son menores a $25,000 / \mu l$.

\section{Pacientes con COVID-19 y trombosis demostrada o fuerte sospecha de TEP}

En pacientes con imagen diagnóstica de TEP, y en aquellos con una fuerte sospecha de TEP (con dímero D elevado y deterioro súbito de la función respiratoria) en quienes el diagnóstico no pueda ser establecido por imagen, la anticoagulación terapéutica con HBPM debe de iniciarse en ausencia de contraindicaciones.

Debe hacerse lo posible por descartar objetivamente la trombosis mediante una imagen de tomografía de tórax de alta resolución, aunque esto puede ser difícil en algunos casos por la gravedad de los pacientes, la disponibilidad de la sala de tomografía y el peligro para el personal de salud. Alternativamente una ecografía Doppler de los miembros inferiores puede realizarse para buscar trombosis venosa profunda (TVP). El ecocardiograma también puede ayudar, buscando datos de insuficiencia cardiaca derecha. Si se demuestra trombosis, la duración de la anticoagulación debe ser la estándar, con una duración mínima de tres meses.

\section{Pacientes con COVID-19 que egresan del hospital}

Los pacientes egresados de la UCI por neumonía o sepsis tienen hasta cinco veces más riesgo de tener un evento de TEV en las primeras tres semanas del egreso hospitalario. La tromboprofilaxis con HBPM o con DOAC sí reduce el riesgo de trombosis, pero a costa de un incremento en el riesgo de sangrados. Sin embargo, si se escoge al paciente adecuado, como aquel con mayor riesgo trombótico (medido con la escala IMPROVE TVE), con dímero D mayor a dos veces lo normal y con bajo riesgo para sangrar, el beneficio en prevención de la trombosis puede superar el riesgo de sangrados. Para este tipo de pacientes se ha aprobado la tromboprofilaxis extendida con HBPM, betrixabán o rivaroxabán, por una duración entre dos y seis semanas.

\section{Pacientes con COVID-19 grave que egresan DEL HOSPITAL}

Se recomienda la tromboprofilaxis extendida con HBPM o DOAC al menos por dos semanas y hasta 
por 30 o 45 días en el caso de que haya persistencia de factores de riesgo para trombosis (como movilidad reducida, cáncer activo o alguna otra situación protrombótica) o de que el dímero $D$ persista elevado por arriba de dos veces el valor normal.

La experiencia clínica en COVID-19 sugiere que hay fallas de anticoagulación en pacientes que ya reciben anticoagulación profiláctica. En un estudio publicado recientemente, a pesar de la tromboprofilaxis sistemática, el $31 \%$ de los 184 pacientes de las unidades de cuidados críticos con COVID-19 desarrollaron complicaciones trombóticas. En una actualización de esta cohorte, la incidencia acumulada de tromboembolia arterial y venosa fue del $49 \%$ (intervalo de confianza del 95\%: 41-57\%). Otros estudios han encontrado un aumento similarmente marcado en la incidencia de TEV en pacientes que requieren atención en la $\mathrm{UCl}$ que excede a los pacientes de la UCI con cuidados similares sin COVID-19, incluyendo la comparación con pacientes de gripe anteriores. Esto podría deberse a varias razones, entre ellas:

- Cambios en la coagulación específica de COVID-19 con una amplia activación de la coagulación para la cual la dosis profiláctica puede ser insuficiente.

- Ya se había desarrollado una tromboembolia antes de iniciar la anticoagulación.

- Una dosificación inadecuada (alto índice de masa corporal o dosificación inexacta).

En el entorno de los pacientes críticamente enfermos que requieren ventilación mecánica puede producirse una inmovilización debido a la sedación profunda y a la parálisis muscular.

Además, el uso de ajustes de ventilación de alta presión que dan lugar a una alta presión intratorácica puede perjudicar la perfusión pulmonar. Se ha desarrollado una controversia significativa en relación con un aumento empírico de la dosis de heparina, especialmente en las siguientes situaciones:

- Empeoramiento del cuadro clínico, que se manifiesta en un aumento de las necesidades de oxígeno, que puede deberse a los microtrombos pulmonares.

- Sospecha de TEP basada en el desarrollo abrupto de hipoxemia, nueva taquicardia y tensión del corazón derecho observada en el ecocardiograma.

- Necesidad de cuidados en la UCI y apoyo ventilatorio, ya que se ha descubierto que la incidencia acumulada de TEV en los pacientes de la UCI es mayor que en los que no requieren cuidados en la $\mathrm{UCl}^{24,25}$.

\section{Trombocitopenia Trombótica asociada a vacunación (TTAV)}

Las vacunas son fundamentales para contener eficazmente la COVID-19. Cuatro vacunas han sido aprobadas por la Agencia Médica Europea (EMA) hasta 30 de marzo de 2021: dos vacunas basadas en ARNm que codifican la proteína de pico del síndrome respiratorio coronavirus 2 (SARS-CoV-2) de Pfizer/ BioNTech y Moderna, y dos vacunas basadas en vectores adenovirales recombinantes que codifican la proteína de pico de AstraZeneca/Universidad de Oxford y Janssen/Johnson \& Johnson. Más de 120 millones de dosis de la vacuna había sido administrada en Europa (https://ourworldindata.org/COVIDvaccinations) a 31 de marzo de 2021. A pesar de los esfuerzos científicos e industriales sin precedentes, el suministro de vacunas aún no satisface la demanda urgente de alcanzar la inmunidad protectora en la población general en aumento a presión para utilizar todas las vacunas aprobadas.

Más de 20 millones de dosis de la vacuna COVID19 AstraZeneca (AZD1222, ChAdOx1 nCoV-19) se han administrado en países de la Unión Europea y el Reino Unido antes del 25 de marzo de $2021^{26}$. Sin embargo, informes recientes de trombosis grave del seno venoso cerebral (CVST) poco común después de la vacunación han provocado la suspensión temporal de la vacuna en 16 continentes. El 26 de marzo de 2021, la EMA autorizó el uso adicional de la vacuna COVID-19 de AstraZeneca, que pasó a llamarse Vaxzevria ${ }^{27}$. La VITT fue descrita por primera vez por grupos en Noruega y Alemania entre los receptores de la vacuna ChAdOx1 en esos países, lo que provocó el cese temporal de la vacuna dentro de la Unión Europea. Esto fue seguido pronto por una serie más grande del Reino Unido que definió aspectos clínicos. de VITT y su diagnóstico de laboratorio.

El VITT es un síndrome poco común que afecta aproximadamente a una persona de cada 50.000 a 100.000 personas vacunadas con ChAdOx1. Los pacientes presentan síntomas graves entre 5 y 30 días después de la vacunación, excepto en algunos casos con trombosis venosa profunda aislada y embolia pulmonar, donde la presentación es hasta 42 días después de la vacunación. La aparición de VITT como un evento adverso raro pero grave que complica la vacunación COVID-19 impulsó una acción urgente de la comunidad hematológica que resultó en la rápida formación de un Panel de Expertos en Hematología (EHP) para asesorar sobre la 
investigación y el manejo de más de 300 casos potenciales de VITT ${ }^{28}$. A partir de julio del 2021 la Organización Mundial de la Salud (OMS), ha publicado unas guías de diagnóstico y tratamiento para la trombocitopenia trombótica postvacunación, esto debido al número creciente de casos) ${ }^{29}$, que inicialmente insinúa efectos específicos de los lotes relacionados con los componentes de las vacunas adenovirales. Más recientemente, una actualización del gobierno del Reino Unido hasta el 21 de marzo documentó 22 informes de CVST y 8 informes de otros eventos de trombosis con recuentos bajos de plaquetas $^{30}$. A 4 de abril de 2021, se notificaron un total de 169 casos de CVST y 53 casos de trombosis de la vena esplácnica a EudraVigilance ${ }^{31}$. Alrededor de 34 millones de personas se habían vacunado con Vaxzevria en el Reino Unido para esta fecha. El 7 de abril, el comité de seguridad de la EMA (PRAC) llegó a la conclusión de que los trombos con recuentos bajos de plaquetas deben incluirse como efectos secundarios muy raros de Vaxzevria ${ }^{31}$.

Andreas Greinacher, et al., en la Universidad de Greifswald, establecieron el mecanismo patogénico posible que subyace a estos raros incidentes de venas, principalmente intracraneales, trombosis asociadas con trombocitopenia, a las que llamaron trombocitopenia protrombótica inmune inducida por vacuna (VIPIT/VITT) ${ }^{32}$. El paciente índice fue una mujer con una trombosis esplácnica seguido de una trombosis aórtica y venosa cerebral poco después la primera vacuna, y se documentó una serie de ocho casos similares (7 mujeres, 1 hombre), siete presentaron trombosis de la vena cerebral y uno embolia pulmonar. Todos tenían concomitantemente trombocitopenia $(13,000-100,000 / \mu \mathrm{l})$. Los primeros síntomas se presentaron de 4 a 16 días después de la vacunación, fallecieron cuatro pacientes. En cuatro pacientes se documentaron anticuerpos dirigidos contra el factor 4 plaquetario (PF4)/heparina y se encontraron complejos y estos sueros activaron plaquetas de prueba lavadas de donantes normales. Por lo tanto, los hallazgos de laboratorio en estos raros casos después de la vacunación con Vaxzevria se parecían a la HIT, un trastorno protrombótico trombocitopénico causado por la formación de inmunoglobulina $\mathrm{G}(\lg G)$ contra nuevos epítopos expuestos después de la asociación de heparina u otros polianiones con factor plaquetario 4 (PF4, CXCL4) secretados por las plaquetas $^{8}$. Por sus dominios Fc, estos complejos inmunes se unen a FcyRIIA en la superficie de las
Tabla 2. Actividad anti Xa para las heparinas

\begin{tabular}{|l|l|l|}
\hline & $\begin{array}{l}\text { Heparina no } \\
\text { fraccionada }\end{array}$ & Heparina de bajo peso molecular \\
\hline $\begin{array}{l}\text { Dosis } \\
\text { profiláctica }\end{array}$ & $0.10-0.15 \mathrm{UI} / \mathrm{ml}$ & $0.4-0.6 \mathrm{UI} / \mathrm{ml}$ \\
\hline $\begin{array}{l}\text { Dosis } \\
\text { terapéutica }\end{array}$ & $0.3-0.7 \mathrm{UI} / \mathrm{ml}$ & $\begin{array}{l}0.6-1 \mathrm{UI} / \mathrm{ml} \text { (para aplicación cada } \\
12 \mathrm{~h}) \\
1-1.5 \mathrm{UI} / \mathrm{ml} \text { (para aplicación cada } \\
24 \mathrm{~h})\end{array}$ \\
\hline
\end{tabular}

Tabla 3. Vigilancia de la hemostasia en los pacientes con COVID-19

\begin{tabular}{|c|c|c|}
\hline Indicación & Pruebas & Comentario \\
\hline $\begin{array}{l}\text { Monitoreo básico } \\
\text { de la coagulación }\end{array}$ & $\begin{array}{l}\text { Biometría } \\
\text { (Plaquetas) } \\
\text { TP } \\
\text { Fibrinógeno } \\
\text { Dímero D }\end{array}$ & $\begin{array}{l}\text { Diario, o al menos cada } \\
2-3 \text { días }\end{array}$ \\
\hline $\begin{array}{l}\text { Pacientes con } \\
\text { heparinas }\end{array}$ & $\begin{array}{l}\text { TTPa } \\
\text { Creatinina } \\
\text { Actividad anti } \\
\text { Xa }\end{array}$ & $\begin{array}{l}\text { Pacientes con insuficiencia } \\
\text { renal, obesidad o estado } \\
\text { crítico }\end{array}$ \\
\hline $\begin{array}{l}\text { Pacientes con } \\
\text { resistencia a la } \\
\text { heparina }\end{array}$ & Antitrombina & $\begin{array}{l}\text { La antitrombina puede bajar } \\
\text { si hay CID o hepatopatía }\end{array}$ \\
\hline $\begin{array}{l}\text { Situaciones } \\
\text { especiales }\end{array}$ & ROTEM O TEG & En pacientes con sangrado \\
\hline
\end{tabular}

Tabla 4. Dosis intermedias de las heparinas

\begin{tabular}{|l|c|c|}
\hline Enoxaparina & Nadroparina & Heparina no fraccionada \\
\hline $60 \mathrm{mg}$ cada $24 \mathrm{~h}$ & $5,700 \mathrm{Ul}$ cada $24 \mathrm{~h}$ & $7,500 \mathrm{Ul}$ cada $8 \mathrm{~h}$ \\
\hline $40 \mathrm{mg}$ cada $12 \mathrm{~h}$ & $3,800 \mathrm{Ul}$ cada $12 \mathrm{~h}$ & \\
\hline
\end{tabular}

plaquetas, por lo que se entrecruzan estos receptores e inducen la activación plaquetaria ${ }^{8,9}$.

Los sueros fueron inhibidos por altas concentraciones de heparina o FcyRIIA que protegía a $\operatorname{lgG}^{32,33}$.

La estimulación del FcyRIIA plaquetario conduce a la activación río abajo de la tirosina cinasa de Bruton $(B t k)^{33}$ como un factor decisivo vía de señalización para los pasos posteriores de la activación plaquetaria. Recientemente hemos demostrado que la activación plaquetaria (incluida la agregación, la secreción de gránulos densos y la expresión de P-selectina), así como la formación de agregados plaquetas-neutrófilos estimulados por reticulación de FcyRIIA o sueros de pacientes con HIT, se suprimió por completo incubando sangre con bajas concentraciones de varios inhibidores de Btk (BTKi) in vitro ${ }^{34}$. 
Tabla 5. Recomendaciones para el diagnóstico y tratamiento de VIPIT. Es particularmente importante la clínica con el antecedente de vacunación

Recomendaciones de diagnóstico y tratamiento de VITT

Trombosis 5 a 28 días posvacunación (Astra Zeneca o Johnson and Johnson)

Trombosis en sitios poco usuales, por ejemplo:

- Trombosis en seno cerebral (50\%)

- Trombosis en vena esplácnica

- Isquemia arterial

- Trombosis venosa profunda o embolia pulmonar

Edad: 19-84 años (media: 45)

Sexo: hombre/mujer 50/50

Trombocitopenia $\left(<150 \times 10^{9} /\right)$

Elevación de dímeros $\mathrm{D}(>4,000 \mathrm{ng} / \mathrm{ml})$

Disminución en la concentración de fibrinógeno

Prueba de Elisa para (HIT) positiva (anticuerpos antifactor

4 plaquetario)

Constituye una urgencia de tratamiento:

- Administrar lgG intravenosa en altas dosis (1 g x kg/día 2 días)

- Anticoagulación con fondaparinux o anticoagulantes orales directos (ACOD) (con cifra de plaquetas $>30 \times 10^{9} /$ )

- No transfundir plaquetas

- No emplear heparinas

En caso de no respuesta valorar recambio plasmático

VIPIT: trombocitopenia protrombótica inmune inducida por vacuna; HIT: trombocitopenia inducida por heparina: Ig: inmunoglobulina.

La patogenia de HIT y obviamente VIPIT también involucra a otras células además de plaquetas: monocitos, neutrófilos y células endoteliales. Los BTKi suprimieron la expresión de P-selectina en las plaquetas, que participan de manera crucial en su interacción con los monocitos para promover la expresión del factor tisular amplificando la formación de trombina. Los BTKi también inhiben la estimulación de monocitos y neutrófilos mediada por FcyRIIA ${ }^{33}$. La acumulación de neutrófilos y la liberación de trampa extracelular de neutrófilos (NET) contribuyen a la trombosis en HIT y HIT.

Los complejos inmunes inducen la liberación de NET por medio de la interacción con FçRIIA en neutrófilos y la asociación neutrófilo-plaquetas inhibida por BTKi ${ }^{34}$. Además, la inhibición de linfocitos B por BTKi se espera que reduzca la producción de anticuerpos patógenos anti-PF4. En general, estos hallazgos establecen una plétora de mecanismos deletéreos más allá de las plaquetas. La activación podría ser dirigida pleiotrópicamente por BTKi en la patogénesis de VITT.
Mientras que en la COVID-19 los signos de una disposición protrombótica general están bien documentados, la localización predominante poco común de trombosis en las venas del seno cerebral en VIPIT es misterioso. Un factor de localización adicional parece estar funcionando. En la infección por SARSCoV-2 puede ocurrir neuroinvasión y la proteína de punta está involucrada ${ }^{28}$, pero la CVST no ha surgido como un subconjunto prominente en las manifestaciones neurológicas heterogéneas ${ }^{34}$.

El endotelio dañado podría unirse a PF4-IgG libre y/o unido a plaquetas circulante complejos en pacientes propensos a VITT. Recientemente se ha demostrado que el PF4 se une en varios sitios a lo largo de la superficie de cadenas extendidas de FvW liberado del endotelio después de una lesión fotoquímica ${ }^{30}$. Los complejos PF4/FvW fueron reconocidos por anticuerpos de pacientes con HIT y promovió la adhesión de plaquetas y el crecimiento de trombos bajo flujo. En particular, la adhesión de plaquetas a los complejos de anticuerpos PF4-FvW-HIT fue inhibida por anticuerpos que bloquearon no solo FcyRIIA sino también el complejo GPIb-IX en las plaquetas.

Curiosamente, la estimulación de GPIb por el FvW también activa la Btk ${ }^{31}$, y el BTKi reduce la activación plaquetaria en sangre mediada por FvW/GPIb (medida por agregación inducida por ristocetina).

Los BTKi aprobados ahora se utilizan ampliamente como estándar medicamentos para la terapia oral a largo plazo de varias neoplasias malignas de células B con una seguridad notable perfil y ejerció un efecto aparentemente protector en caso de coincidencia sintomática COVID-1930-33. Cabe destacar que las concentraciones inhibidoras de plaquetas de los BTKi aprobados (ibrutinib, acalabrutinib, zanubrutinib y tirabrutinib) fueron mucho más bajas que los niveles alcanzados en pacientes tratados con dosis estándar orales para trastornos de células $B$.

Además, la ingesta de una dosis única de ibrutinib (280 mg) por voluntarios humanos sanos rápidamente bloqueado ( 3 horas después de la ingesta) la agregación y secreción de plaquetas en la estimulación máxima de FcyRIIA en plaquetas en sangre ex vivo. La inhibición plaquetaria selectiva de estímulo/receptor fue sostenido durante hasta dos días, lo que se explica por la unión covalente de ibrutinib a Btk y la falta de síntesis de proteínas de novo en plaquetas. Supresión de la activación plaquetaria mediada por Btk. Se ha demostrado previamente que se mantiene con una dosis baja de ibrutinib (140 mg por día o en días alternos). 


\section{Conclusiones}

Los pacientes de COVID-19 en tratamiento de tromboprofilaxis con heparina no solo presentan hipercoagulabilidad, sino también fibrinólisis deficiente que, en conjunto, pueden contribuir a un riesgo de trombosis en pacientes que reciben un tratamiento antitrombótico. Los estudios futuros deberían evaluar la eficacia y la seguridad de las dosis escalonadas de heparina en pacientes con COVID-19. Por otro lado, ha surgido la asociación entre la vacuna de Astra Zeneca y casos de trombosis y trombocitopenia, es importante que en los países que se administre esta vacuna o la de Johnson \& Johnson vigilen el cuadro de VITT y efectuar un tratamiento oportuno (Tabla 5).

\section{Financiamiento}

No se recibió financiamiento ni patrocinio por ninguna organización o institución.

\section{Conflicto de intereses}

Los autores declaran no tener conflicto de intereses alguno con el tema de la publicación.

\section{Responsabilidades éticas}

Protección de personas y animales. Los autores declaran que para esta investigación no se han realizado experimentos en seres humanos $\mathrm{ni}$ en animales.

Confidencialidad de los datos. Los autores declaran que en este artículo no aparecen datos de pacientes.

Derecho a la privacidad y consentimiento informado. Los autores declaran que en este artículo no aparecen datos de pacientes.

\section{Bibliografía}

1. Yin S, Huang M, Li D, Tang N. Difference of coagulation features between severe pneumonia induced by SARS-CoV2 and non-SARS-CoV2. J Thromb Thrombolysis. 2021;51(4):1107-10.

2. Tang N, Bai H, Chen X, Gong J, Li D, Sun Z. Anticoagulant treatment is associated with decreased mortality in severe coronavirus disease 2019 patients with coagulopathy. J Thromb Haemost. 2020;18(5):1094-9.

3. Llitjos JF, Leclerc M, Chochois C, Monsallier JM, Ramakers M, Auvray M, et al. High incidence of venous thromboembolic events in anticoagulated severe COVID-19 patients. J Thromb Haemost. 2020;18(7):1743-6.

4. Klok FA, Kruip MJHA, van der Meer NJM, Arbous MS, Gommers DAM PJ, Kant KM, et al. Incidence of thrombotic complications in critically ill ICU patients with COVID-19. Thromb Res. 2020;191:145-7.

5. Connors JM, Levy JH. COVID-19 and Its Implications for thrombosis and anticoagulation. Blood. 2020;135(23):2033-40.
6. Tang N, Li D, Wang X, Sun Z. Abnormal coagulation parameters are associated with poor prognosis in patients with novel coronavirus pneumonia. J Thromb Haemost. 2020;18(4):844-7.

7. Zhang Y, Xiao M, Zhang S, Xia P, Cao W, Jiang W, et al. Coagulopathy and antiphospholipid antibodies in patients with COVID-19. N Engl J Med. 2020;382(17):e38.

8. de Bruijne EL, Gils A, Guimarães AH, Dippel DW, Deckers JW, van den Meiracker $\mathrm{AH}$, et al. The role of thrombin activatable fibrinolysis inhibitor in arterial thrombosis at a young age: the ATTAC study. J Thromb Haemost. 2009;7(6):919-27.

9. Tregouet DA, Schnabel R, Alessi MC, Godefroy T, Declerck PJ, Nicaud V, et al.; AtheroGene Investigators. Activated thrombin activatable fibrinolysis inhibitor levels are associated with the risk of cardiovascular death in patients with coronary artery disease: The AtheroGene Study. J Thromb Haemost. 2009;7:49-57.

10. Redlitz A, Nicolini FA, Malycky JL, Topol EJ, Plow EF. Inducible carboxypeptidase activity. A role in clot lysis in vivo. Circulation. 1996:93:1328-30.

11. Meltzer ME, Lisman T, de Groot PG, Meijers JC, le Cessie S, Doggen CJ, et al. Venous thrombosis risk associated with plasma hypofibrinolysis is explained by elevated plasma levels of TAFI and PAI-1. Blood. 2010;116(1):113-21.

12. Prabhakaran P, Ware LB, White KE, Cross MT, Matthay MA, OIman MA. Elevated levels of plasminogen activator inhibitor-1 in pulmonary edema fluid are associated with mortality in acute lung injury. Am J Physiol Lung Cell Mol Physiol. 2003;285:L20-8.

13. Longstaff C. Measuring Fibrinolysis. Hämostaseologie. 2021;41:69-75.

14. Whiting D, DiNardo JA. TEG and ROTEM: technology and clinical applications. Am J Hematol. 2014;89(2):228-32.

15. Panigada M, Bottino N, Tagliabue P, Grasselli G, Novembrino C, Chantarangkul V, et al. Hypercoagulability of COVID-19 patients in Intensive Care Unit. A Report of Thromboelastography Findings and other Parameters of Hemostasis. J Thromb Haemost. 2020;18(7):1738-42.

16. Spiezia L, Boscolo A, Poletto F, Cerruti L, Tiberio I, Campello E, et al. COVID-19-related severe hypercoagulability in patients admitted to intensive care unit for acute respiratory failure. Thromb Haemost. 2020;120(6):998-1000.

17. Thachil J, Tang N, Gando S, Falanga A, Cattaneo M, Levi M, et al. ISTH interim guidance on recognition and management of coagulopathy in COVID 19. J Thromb Haemost. 2020;18(5):1023-6.

18. Helms J, Tacquard C, Severac F, Leonard-Lorant I, Ohana M, Delabranche X, et al.; CRICS TRIGGERSEP Group (Clinical Research in Intensive Care and Sepsis Trial Group for Global Evaluation and Research in Sepsis). High risk of thrombosis in patients with severe SARS-CoV-2 infection: a multicenter prospective cohort study. Intensive Care Med. 2020;46(6):1089-8.

19. Xu Z, Shi L, Wang Y, Zhang J, Huang L, Zhang C, et al. Pathological findings of COVID-19 associated with acute respiratory distress syndrome. Lancet Respir Med. 2020;8(4):420-2.

20. Klok FA, Kruip MJHA, van der Meer NJM, Arbous MS, Gommers DAMPJ, Kant KM, et al. Incidence of thrombotic complications in critically ill ICU patients with COVID-19. Thromb Res. 2020;191:145-7.

21. Bikdeli B, Madhavan MV, Gupta A, Jimenez D, Burton JR, Der Nigoghossian $\mathrm{C}$, et al. Pharmacological agents targeting thromboinflammation in COVID-19: Review and implications for future research. Thromb Haemost. 2020;120(7):1004-24.

22. Obi AT, MD, Barnes GD, Napolitano LM, MD, Henke PK,Wakefield TW. Venous thrombosis epidemiology, pathophysiology, andanticoagulant therapies and trials in severe acute respiratory syndrome coronavirus 2 infection. J Vasc Surg Venous Lymphat Disord. 2021:9(1):23-35.

23. Cattaneo M, Bertinato EM, Birocchi S, Brizio C, Malavolta D, Manzoni M, et al. Pulmonary embolism or pulmonary thrombosis in COVID-19? Is the recommendation to use high-dose heparin for thromboprophylaxis justified? Thromb Haemost. 2020;120(8):1230-2.

24. Moonla Ch, Sosothikul D, Chiasakul T, Rojnuckarin P, Uaprasert N. Anticoagulation and in-hospital mortality from coronavirus disease 2019: A systematic review and meta-analysis. Clin Appl Thromb Hemost. 2021;27:10760296211008999.

25. von Hundelshausen P, Lorenz R, Siess W, Weber C. Vaccine-induced immune thrombotic thrombocytopenia (VITT): targeting pathomechanisms with Bruton tyrosine kinase inhibitors. Thromb Haemost. 2021 Apr 13. doi: 10.1055/a-1481-3039. Online ahead of print.

26. Callaway E, Mallapaty S. What scientists do and don't know about the oxford-astrazeneca COVID vaccine. Nature. 2021;592:15-7.

27. European Medicines Agency. Vaxzevria1 (COVID-19 vaccine (ChAdOx1 -s [recombinant])) Report No.: EMA/182334/2021, EMEA/H/C/005675 [Internet]. European Medicines Agency; 26 marzo 2021. Disponible en: https://www.ema.europa.eu/en/documents/overview/vaxzevria-previously-covid-19-vaccine-astrazeneca-epar-medicine-overview_en.pdf 
28. Chevassut T, Hunt BJ, Pavord S. VITT, COVID-19 and the Expert Haematology Panel: The story of how the UK responded to emerging cases of vaccine-induced immune thrombocytopenia and thrombosis during the vaccination programme. Clin Med (Lond). 2021:2021-0488. doi: 10.7861/ clinmed.2021-0488.

29. Greinacher A, Langer $F$, Makris M, Pai M, Pavord S, Tran $H$, et al Vaccine-induced immune thrombotic thrombocytopenia (VITT): Update on diagnosis and management considering different resources. J Thromb Haemost. 2021. doi: 10.1111/jth.15572. Disponible en: https://onlinelibrary.wiley.com/doi/full/10.1111/jth.15572

30. Gobierno de Reino Unido. Coronavirus vaccine - weekly summary of yellow card reporting [Internet]. Gobierno de Reino Unido [citado: 6 abril 2021]. Disponible en: https://www.gov.uk/government/publications/coronavirus-covid-19-vaccine-adverse-reactions/coronavirus-vaccine-summary-of-yellow-card-reporting
31. European Medicines Agency. EMA confirms overall benefit-risk remains positive [Internet]. European Medicines Agency News; 7 abril 2021. Disponible en: https://www.ema.europa.eu/en/news/astrazenecas-COVID-19-vaccine-ema-findspossible-link-very-rare-cases-unusual-blood-clots-low-blood

32. Greinacher A, Thiele T, Warkentin TE, Weisser K, Kyrle P, Eichinger S. A prothrombotic thrombocytopenic disorder resembling heparin-induced thrombocytopenia following coronavirus-19 vaccination. Research Square [Internet]. 2021:1-8. Disponible en: https://www.researchsquare.com/ article/rs-362354/v1

33. Greinacher A. Clinical practice. Heparin-induced thrombocytopenia. N Engl J Med. 2015;373:252-61.

34. Goldmann L, Duan R, Kragh T, Wittmann G, Weber C, Lorenz R, et al. Oral Bruton tyrosine kinase inhibitors block activation of the platelet Fc receptor CD32a (FcyRlla): A new option in hit? Blood Adv. 2019;3:4021-33. 